\title{
Utilisation des techniques de l'information et de la communication par les étudiants en quatrième année d'études de médecine à la faculté de Strasbourg (France). Evolution sur quatre promotions
}

\author{
Computer Technologies Utilization by fourth Year Medical Students in Strasbourg (France). \\ Improvement on four years
}

François BECMEUR ${ }^{1}$, Michèle KIRCH ${ }^{2}$, Jacques MARESCAUX ${ }^{3}$, Didier MUTTER ${ }^{3}$

Résumé Contexte : Les technologies de l'information et de la communication (TIC) sont devenues une source d'information habituelle chez les étudiants. But : L'objectif de ce travail était de mieux connaître l'évolution de l'accessibilité des TIC et du niveau de compétences dans l'utilisation de l'informatique, chez les étudiants hospitalier en 4' année d'études de médecine dans la faculté de Strasbourg en 5 ans (entre 2001 et 2005). Méthodes : Les étudiants ont répondu à un questionnaire portant sur leur niveau d'équipement en informatique et les modes d'utilisation des TIC. Une question à réponse ouverte portait sur la place et l'utilisation des TIC dans la formation médicale. Résultats : A peine plus d'un étudiant sur deux possède son propre ordinateur, mais $90 \%$ ont un accès facile aux TIC. Les compétences en utilisation de l'outil informatique progressent essentiellement chez les étudiantes. On constate le développement des connexions à haut débit sur Internet et les possibilités de mémoires portables de grande capacité (clé USB). La majorité des étudiants reconnaissent une place progressivement croissante des TIC dans leur formation. Ils font l'analyse critique des contenus existants et disponibles sur Internet pour leur formation. Conclusion: Pour que les TIC deviennent plus qu'une ressource informative mais un contenu pédagogique vrai, il faut que l'équipement informatique des étudiants progresse et que les enseignants améliorent l'usage quills font des TIC à des fins pédagogiques.

Mots clés Technologies de l'information et de la communication ; pédagogie médicale ; équipement informatique des étudiants.

Abstract Context: Medical students routinely use computer technologies dedicated to convey information for learning purposes. Objective: To determine the evolution of computer technologies availabilities and to analyze the progression of the level of competency of computer use by 4 th year medical students at the Faculty of Strasbourg, during a period of five years (2001-2005). Methods: Students have filled a questionnaire raising their computer equipment status and their degree of computer technologies utilization. An open question hoisted the importance of computer technologies for medical formation. Results: In 2005, hardly more than the half of students owns a personal computer, although 90 per cent of them can access to it easily. The level of competency to use computers has essentially improved for female students. Moreover, for most students, high-speed Internet connections and portable computer memory (USB keys) have become common. The importance of computer technologies for medical student formation was also confirmed. However, it was noted that students frequently criticize existing educational web sites for their lack of content. Conclusion: Computer technologies can become an interesting and reliable pedagogical tool for medical students if there is a progression in student computer ownership and if teachers optimize computer technologies utilization for pedagogical purposes.

Key words Computer technologies; medical education; student's computer equipment.

Pédagogie Médicale 2006;7:43-52 


\section{Nouvelles Technologies Éducatives}

\section{Introduction}

Les technologies de l'information et de la communication (TIC) s'ajoutent aux instruments de transmission du savoir que sont le livre, le polycopié et le cours magistral. Un contenu scientifique est actuellement disponible en masse sur Internet. Il est présent sans intention pédagogique particulière et demeure souvent la simple transposition de documents papiers associés à une imagerie plus ou moins abondante. La mise à disposition de cours sur Internet ne suffit probablement pas à l'apprentissage dès lors qu'elle n'est pas associée à une démarche pédagogique claire. Mais l'interactivité des TIC (liens hypertexte, vidéo, animations, questions-réponses et auto-évaluations) les placent potentiellement parmi les outils destinés à la construction des connaissances comme le sont les travaux dirigés. La place des TIC dans l'enseignement, la notion d'enseignement in abstentia, l'exploration des ressources d'un nouvel outil en vue d'une pédagogie nouvelle font l'objet de nombreux questionnements et travaux ${ }^{1,2,3,4}$.

Les auteurs ont collaboré à une université virtuelle (Websurg.com), cahier des charges et chapitres. Dans le contexte d'équipes multidisciplinaires (informaticiens, dessinateurs, animateurs, spécialistes de l'image, traducteurs, rédacteurs scientifiques), ils ont découvert quelles pouvaient être les caractéristiques d'un enseignement par Internet. Au travers d'entretiens semi-structurés, l'équipe rédactionnelle avait déjà tenté d'appréhender les attentes ainsi que les problèmes rencontrés par des étudiants en médecine face à une proposition d'utilisation des TIC dans leur formation.

Des questions pratiques se posaient ${ }^{5,6}$ : les TIC sont-elles accessibles à tous les étudiants en médecine? Quelles sont les difficultés rencontrées par les étudiants avec l'usage des TIC ?

Le but de cette étude était de connaitre :

a) le niveau d'équipement des étudiants hospitaliers en informatique, leur niveau de compétence dans la manipulation des TIC ;

b) leurs attentes à l'égard des TIC dans l'apprentissage de leur métier, tant les conditions d'efficacité des formations par les TIC en pédagogie universitaire restent encore à évaluer ${ }^{7}$.

\section{Matériel et méthode}

Une enquête par questionnaire (annexe) a été distribuée en 2001, 2002, 2003 et 2005 aux étudiants hospitaliers de quatrième année. Le questionnaire était proposé et rempli sur place, au début d'un enseignement dirigé et remis 10 minutes plus tard à l'enseignant.

Les questions portaient sur le niveau d'équipement en informatique, le niveau d'utilisation d'un ordinateur et ses modalités d'usage. Une question à réponse ouverte autorisait les remarques personnelles à propos de la place et de l'utilisation des TIC dans la formation médicale.

\section{Résultats}

Tous les étudiants interrogés ont répondu, le questionnaire étant distribué en début de séance de travaux dirigés et collecté dix minutes plus tard. L'enquête était justifiée oralement par : le besoin d'amélioration de la distribution et de l'accessibilité de sites d'accès à Internet sur les terrains de stages pour les étudiants à Strasbourg ; le questionnement à l'égard de l'intérêt de véritables universités virtuelles pouvant à terme compléter voire remplacer les polycopiés et certains enseignements dirigés. Parmi 361 étudiants (Tableau 1), 213 filles (59 \%) et 148 garçons (41\%) ont répondu au questionnaire. La moyenne d'âge est de 22 ans.

En 2005, 53 \% des étudiants interrogés possèdent un ordinateur. Ce chiffre est stable depuis le début de ce travail d'enquête en 2001. Si les étudiants ne possèdent pas leur propre ordinateur, ils utilisent celui des parents (78\% d'entre eux), plus rarement celui de la faculté (15\% d'entre eux), ou d'un(e) ami(e) (7 \% d'entre eux). Si l'on tient compte des étudiants qui possèdent un ordinateur ou ayant à disposition celui de leurs parents, on constate que $90 \%$ des étudiants interrogés en 2005 ont à leur disposition, ou d'accès facile, un ordinateur en comparaison des $80 \%$ des étudiants interrogés en 2001. Cette évolution favorable concerne principalement les filles. Lordinateur est le plus souvent un PC (92\%) et rarement un Macintosch (8\%).

En 2001, 56 \% des étudiants estimaient être du niveau

1- Service de Chirurgie Infantile - Hôpitaux Universitaires de Strasbourg - Hôpital de Hautepierre - 67098 Strasbourg Cedex - France

2- Laboratoire des sciences de l'éducation - ULP - 8 rue de l'université - 67000 Strasbourg - France

3- Institut de Recherche contre les Cancers de I'Appareil Digestif - European Institute of TeleSurgery (IRCAD-EITS)

Hôpitaux Universitaires de Strasbourg - 1 Place de l'Hôpital - 67000 Strasbourg - France

Correspondance : François Becmeur - Service de Chirurgie Infantile - Hôpitaux Universitaires de Strasbourg - Hôpital de Hautepierre 67098 Strasbourg Cedex - France - mailto:Francois.Becmeur@chru-strasbourg.fr 
d'un débutant. Ils ne sont plus que $34 \%$ en 2005 . Les étudiants estimant être du niveau d'un initié étaient $32 \%$ en 2001 et $53 \%$ en 2005 . Ceux estimant être du niveau d'un expert étaient $12 \%$ en 2001 et $13 \%$ en 2005 (Tableau 2). Le niveau de compétence en matière d'informatique, auto-évalué par les étudiants, est stable chez les garçons et progresse chez les filles.

Les programmes de traitement de texte sont utilisés par $90 \%$ des étudiants et deux fois plus souvent que les programmes de présentation de diapositives ou que les tableurs.

Les compacts-discs (CD) de formation (Tableau 3) étaient utilisés chez $54 \%$ des étudiants en 2001, $21 \%$ en 2002, $14 \%$ en 2003 . Ils ne sont plus utilisés que par $9 \%$ des étudiants en 2005.

Internet était déjà fréquenté par $80 \%$ des étudiants interrogés en 2001 et par $92 \%$ des étudiants interrogés en 2005. Mais la connexion haut-débit était accessible pour $25 \%$ des étudiants en 2002 et pour $72 \%$ d'entre eux en 2005. Le téléchargement de documents sur clé USB est une pratique courante pour $21 \%$ des étudiants en 2005 (Tableau 4).

Jusqu'en 2002, les étudiants faisaient peu de chose sur Internet hormis du courrier électronique dans près de $60 \%$ des cas. Moteurs de recherche, sites médicaux ou sites de loisirs étaient utilisés dans moins de $50 \%$ des cas. En 2005, le courrier électronique est utilisé par $70 \%$ des étudiants et le chat par $22 \%$ des étudiants. Les sites de loisirs sont visités par $66 \%$ des étudiants, les sites médicaux par $43 \%$ des étudiants, les moteurs de recherche par $75 \%$ des étudiants.

Parmi les 361 questionnaires, près de $20 \%$ des garçons (soit environ $10 \%$ des étudiants) déclarent jouer sur l'ordinateur (répartis également sur les 4 années d'enquête 2001, 2002, 2003, 2005), pour moins de $5 \%$ des filles (toutes en 2005).

Aucun site visité n'est payant. Un tiers des étudiants estime que l'accès aux sites de l'Internet doit rester gratuit. Deux tiers pensent que payer un abonnement moyen de $28 €$ par an pour une université virtuelle serait acceptable. Si $28 \%$ des étudiants préferent apprendre exclusivement en langue française, $38 \%$ pensent pouvoir lire indifféremment en anglais ou en français.

Les commentaires libres des étudiants sont résumés (Tableau 5).

\section{Discussion}

\section{Equipement informatique}

L'incidence de la propriété d'un ordinateur est restée stable en 5 ans chez les étudiants en médecine. L'accession facile à un ordinateur (étudiant propriétaire ou utilisant celui des parents), chez les étudiants en médecine, a progressé au profit des filles entre 2001 et 2005. Cependant, près d'un étudiant sur deux ne possède pas son propre ordinateur en 2005 et n'y a donc pas accès librement toute la semaine. En effet, de nombreux étudiants ne vivent pas chez leurs parents pour des raisons d'éloignement géographique de leur ville d'origine par rapport à la ville universitaire.

Mais les équipements d'accès au réseau Internet ont beaucoup progressé ${ }^{8}$ puisque les connexions à haut débit étaient utilisées par moins de $25 \%$ des étudiants en 2001 et le sont par $72 \%$ des étudiants en 2005. L'utilisation conjointe des clés USB (petits disques durs externes) permet désormais un accès Internet parfaitement différencié en temps et en matériel d'une séance de travail. Elle facilite la diffusion ou l'impression de documents copiés sur Internet. Or, $21 \%$ des étudiants possèdent une clé USB souvent initialement destinée à être un support pour écouter de la musique (baladeur).

\section{Utilisation de l'informatique}

Les débutants en informatique étaient majoritaires en 2001. Il existait alors une forte demande de formation', actuellement proposée seulement en enseignement facultatif (option) dès le PCEM2 (deuxième année des études médicales en France). En 2005, les deux tiers des étudiants sont à l'aise avec les outils informatiques. La progression est encore plus évidente si l'on analyse les chiffres dans les cohortes des jeunes femmes étudiantes : $28 \%$ se disaient d'un niveau " expert " ou " initié » en 2001, alors qu'elles sont $78 \%$ dans cette situation en 2005. De plus, si en moyenne $10 \%$ des étudiants déclaraient jouer sur l'ordinateur en 2001, 2002 et 2003, il s'agissait exclusivement de garçons. Désormais, en 2005, $14 \%$ des étudiants déclarent jouer avec l'ordinateur et il s'agit, pour plus de la moitié, de jeunes femmes.

Les CD de formation semblent avoir perdu tout attrait auprès des étudiants puisqu'ils étaient utilisés par $54 \%$ d'entre eux en 2001, mais $9 \%$ en 2005. Les propositions de CD gratuits sont plus rares. Le temps passé sur Internet pour une auto formation a sensiblement progressé grâce aux connexions à haut débit. Les documents disponibles sur Internet sont pluriels, réactualisés et gratuits. Un CD demeure un document fixé définitivement, tel un livre en attente d'une possible réédition et il apporte une information univoque au sens propre du terme.

Si le courrier électronique et le chat sont des activités très fréquentes, la visite de sites médicaux ou l'utilisation des moteurs de recherche sont désormais habituelles.

Cependant, le temps passé en recherche d'information 


\section{Nouvelles Technologies Éducatives}

Tableau 1 :

Répartition des effectifs d'étudiants interrogés dans le cadre de l'étude selon les années

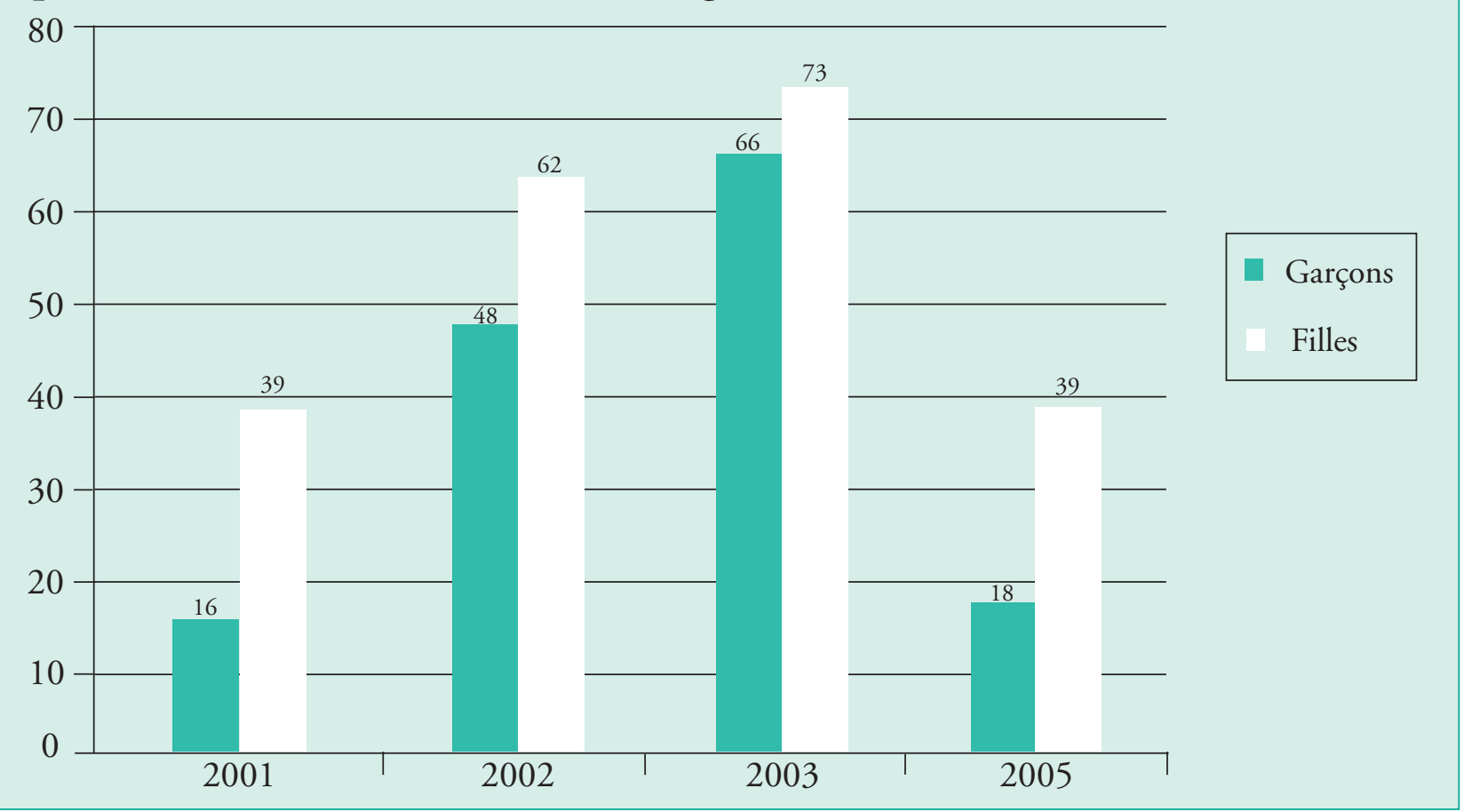

Tableau 2 :

Evolution du niveau de compétence perçu (ou auto-évalué) en informatique, chez les étudiants interrogés, à l'égard des technologies de l'information et de la communication (TIC)

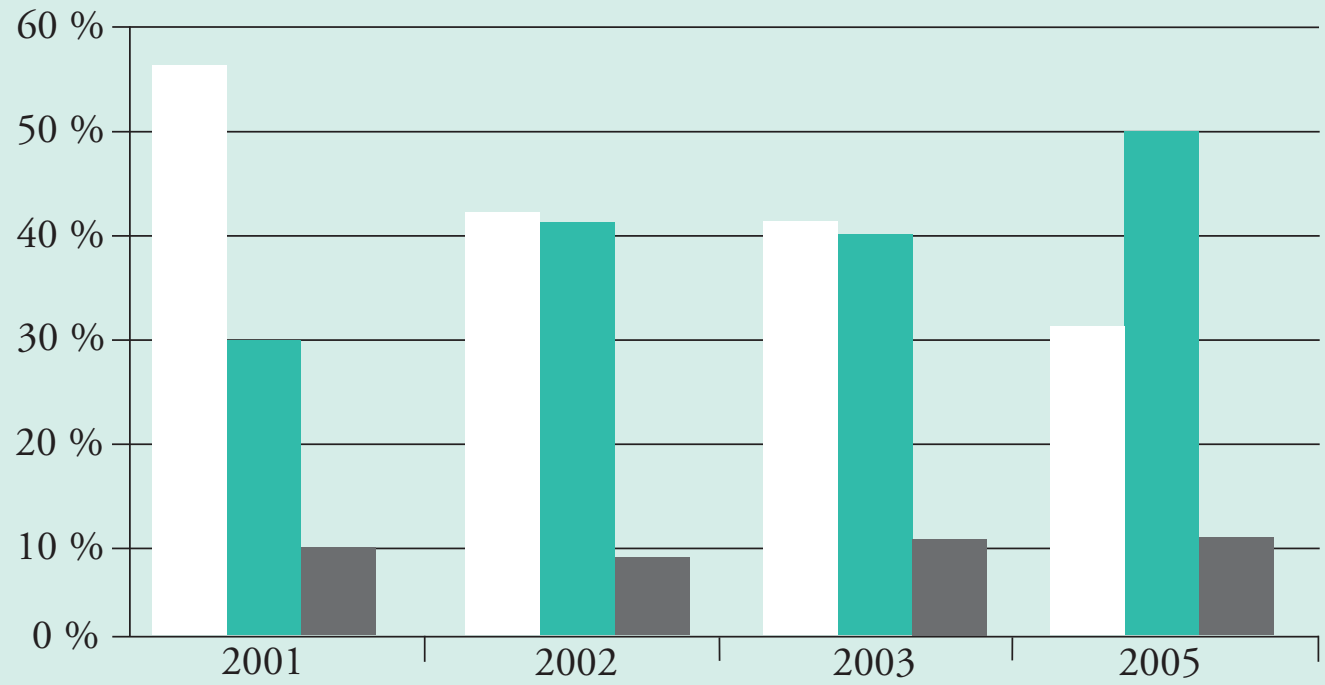

Débutant
Initié
Expert

Les étudiants sont de plus en plus aptes à utiliser les TIC sans rencontrer de difficultés techniques insurmontables ou trop cconsommatrices de temps 
Tableau 3 :

Evolution de l'utilisation des compacts-discs (CD) de formation

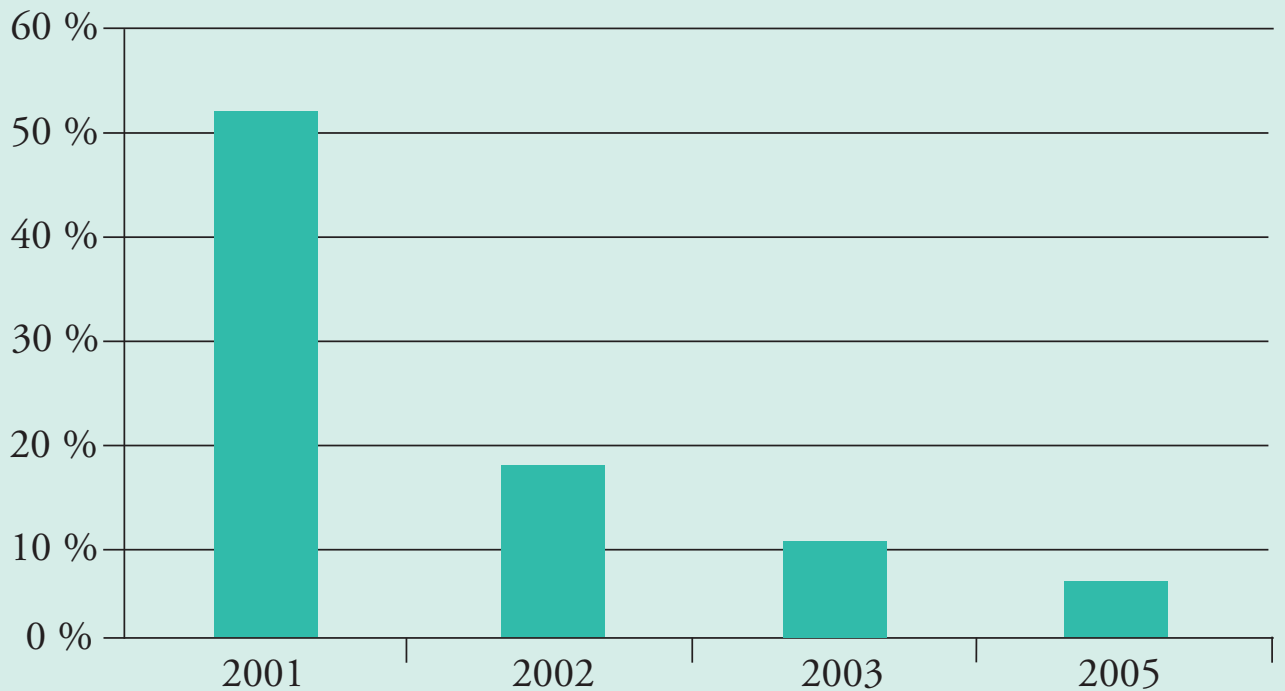

En 2001, $54 \%$ des étudiants disaient avoir eu recours au moins une fois à un CD de formation. Ils ne sont plus que $9 \%$ en 2005, probablement du fait de la meilleure connexion à Internet et de l'utilisation de supports de transfert de données comme la clé USB (21\% des étudiants affirment l'utiliser en 2005).

Tableau 4 :

Evolution des possibilités d'accès à des connexions à haut débit chez les étudiants interrogés

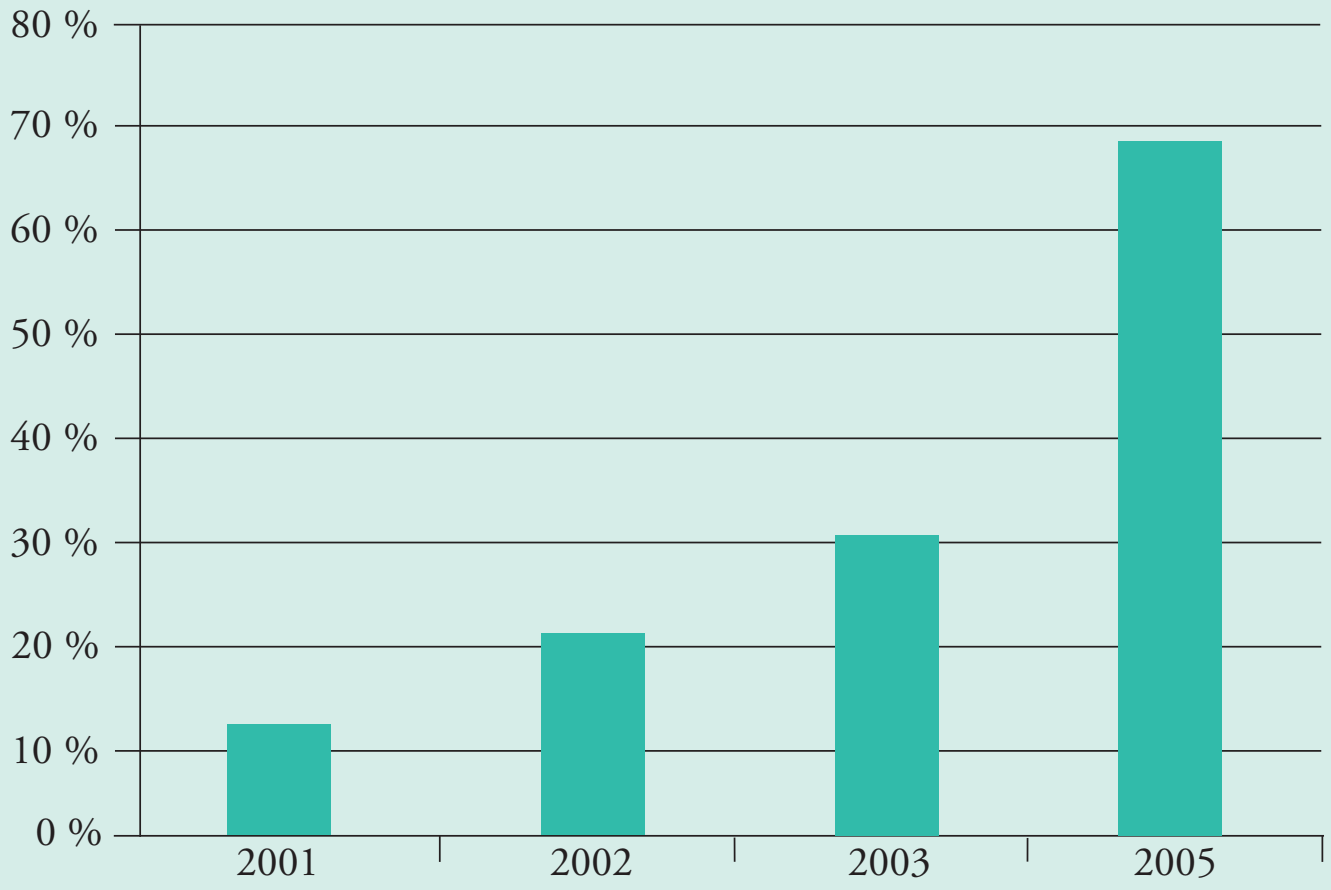




\section{Nouvelles Technologies Éducatives}

\begin{tabular}{|c|c|}
\hline \multicolumn{2}{|r|}{$\begin{array}{l}\text { Tableau } 5 \text { : } \\
\text { Les commentaires libres des étudiants à propos des sites Internet de formation médicale }\end{array}$} \\
\hline 1 & $\begin{array}{l}\text { L'intention pédagogique des sites de formation } \\
\text { - l'enseignement électronique doit faire du neuf } \\
\text { - pas seulement du polycopié en ligne }\end{array}$ \\
\hline 2 & $\begin{array}{l}\text { Les sites de formation doivent avoir la confiance du lecteur } \\
\qquad \begin{array}{l}\text { - crédibilité } \\
\text { - recommandé par un tuteur } \\
\text { - signature (auteur, institution) } \\
\text { - aspects techniques (site protégé, par exemple) }\end{array}\end{array}$ \\
\hline 3 & $\begin{array}{l}\text { Les qualités de lecture des sites de formation } \\
\qquad \begin{array}{l}\text { - accessibilité du site } \\
\text { - présentation claire } \\
\text { - page d'accueil non surchargée notamment par des publicités } \\
\text { - un sommaire immédiatement proposé } \\
\text { - un contenu réellement conçu pour des étudiants et } \\
\text { adapté à leur propre besoin en formation } \\
\text { - une didactisation claire de l'enseignement } \\
\text { - possibilité de plusieurs niveaux de lecture (organisation des liens hypertextes) } \\
\text { - les liens : illustrations, animations, vidéos, documentations, références, } \\
\text { accès à d'autres sites de référence } \\
\text { - version abrégée disponible prête à l'impression " pour mémoire » } \\
\text { - interactivité ; auto-évaluation }\end{array}\end{array}$ \\
\hline
\end{tabular}

sur Internet est toujours redouté. Aussi les étudiants demandent-ils d'être conseillés sur des noms de sites ou d'universités virtuelles qui pourraient contribuer à leur formation de façon efficace sans perdre de temps à découvrir les sites appropriés à leur besoin et leur niveau de formation.

\section{Les sites de formation}

Un site de formation tel l'Université médicale virtuelle francophone (UMVF) ${ }^{8}$ nécessite non seulement des enseignants qui vont proposer un contenu, mais aussi des informaticiens et un personnel qualifié pour animer et mettre en page le contenu de façon interactive (exemple : http://www.websurg.com). Il s'agit de constituer une véritable équipe de production mais aussi de financer le projet. En effet, un site de formation est un objet de consommation qui doit disposer d'un budget de lancement et de fonctionnement et qui doit atteindre un public, c'est-à-dire des consommateurs. A l'échelle d'une université ou d'un pays, le projet, à l'ère de la mondialisation, est peu viable. Il peut devenir pérenne à l'échelle d'une communauté d'universités. Ce peut être à l'échelle de l'Europe (mais en anglais) ou à l'échelle d'une communauté de langue (communauté francophone par exemple). Le financement du site peut dépendre d'un soutien industriel (sponsoring) comme c'est le cas pour http://www.websurg.com. Il peut venir des consommateurs eux-mêmes, individus ou universités, comme c'est le cas pour les sites de journaux médicaux qui requièrent l'abonnement au journal papier (exemples: Journal of Pediatric Surgery, Surgical Endoscopy, etc.) ou bien il peut être lié à des subventions d'état ou d'unions d'états (projet européen E-LERU ${ }^{10}$ ). Le choix de la langue anglaise (anglais scientifique) se heurte à des résistances pour près d'un tiers des étudiants qui préferent apprendre exclusivement en lan- 
gue française. Seulement, $38 \%$ des étudiants pensent pouvoir lire indifféremment en anglais ou en français. De plus, aucun site visité actuellement par les étudiants n'est payant et un tiers des étudiants estime que l'accès aux sites de l'Internet doit rester gratuit.

\section{Commentaires libres des étudiants}

De nombreux étudiants se demandent si l'enseignement électronique a de l'avenir et si les TIC ne servent qu'à accumuler un plus grand savoir (de nombreuses données écrites) dans un plus petit volume. Ils regrettent que le dispositif ne soit pas mieux exploité sur le plan pédagogique. Avoir de plus gros polycopiés sous forme électronique ne leur semble pas être l'enjeu de l'enseignement électronique. L'enseignement électronique doit faire du neuf ${ }^{11}$ et proposer des formules nouvelles, interactives, utilisant toutes les possibilités de l'outil informatique. Les étudiants ne veulent pas étudier exclusivement sur écran pour des raisons pratiques diverses : fatigue, inconfort oculaire, maux de tête, impossibilité d'annoter le texte, disponibilité des TIC, leur coût et la crainte de perte de temps. Ils désirent préserver une interaction directe avec leurs enseignants.

\section{Qu'attendent les étudiants d'un site de formation?}

\section{Le site doit avoir la confiance du lecteur}

Le problème de la crédibilité du contenu des sites Internet est posé. Le texte lu sur Internet est crédité de la confiance $\mathrm{du}$ lecteur dès lors qu'il existe une cohérence avec ses propres connaissances personnelles; les références bibliographiques semblent indispensables. La « signature » du chapitre étudié sur le site, c'est-à-dire le nom de l'auteur, apparaît être déjà « en soi » une référence. Lorsque l'auteur est déjà connu de l'étudiant, il bénéficie $a$ priori de sa confiance. Stanley Milgram ${ }^{12}$ parlait d'autorité reconnue. De la même façon, si le site Internet visité émane d'une faculté ou d'une institution reconnue, il obtient a priori la confiance de l'étudiant. La présence d'une photo de l'auteur est signalée par les étudiants comme un élément favorable de " reconnaissance " qui vient rompre l'anonymat relatif de l'auteur aux yeux de l'étudiant. Enfin la crédibilité peut être d'ordre technique, purement informatique : un site " protégé " par un accès limité avec mot de passe semble apporter du crédit au contenu.

\section{Un site de formation doit être facile à lire}

Les critères de choix d'un site Internet regroupent : l'accès facile du site, une bonne présentation dont les caractéristiques seront définies plus loin, un contenu qui paraît com- plet, la réputation du site et de l'auteur du chapitre, la présence de vidéos et d'animations ou illustrations en quantité suffisante, la clarté du sommaire qui donne envie d'aller plus loin. Certains étudiants souhaitent que le site "soit réellement destiné aux étudiants". Ce besoin est probablement ressenti par habitude de didactisation des savoirs chez de jeunes étudiants tout juste sortis de la période scolaire.

La présentation du site semble avoir une grande importance et les notions d'esthétique et de plaisir de la lecture sont volontiers évoquées. La clarté, la concision du texte, et la possibilité d'avoir en début et fin de chapitre une vue d'ensemble synthétique sont des éléments fréquemment cités. Cependant, l'apparence du site (couleur, richesse des images, animations) ne doit pas faire obstacle techniquement à la lecture du chapitre : si le délai de téléchargement de chaque page est trop important, le site ne pourra pas être lu de façon aisée. L'aspect trop chargé de la page d'accueil d'un site, ou une lecture trop complexe ou confuse, l'existence de publicités avant d'accéder à l'objet de la visite font rebrousser chemin. Le caractère ludique d'un site trop pourvu en animations ou liens est perçu comme exposant au risque de perdre du temps et de perdre le fil de la lecture, s'opposant ainsi à la vocation pédagogique revendiquée par ces outils. Comme si trop de pédagogie tuait la pédagogie. Le maître mot de l'apparence est la clarté offrant une vue d'ensemble synthétique.

\section{Le texte}

Le texte doit être lisible. Ce qui semble de prime abord être une évidence correspond à un véritable problème de lecture sur écran informatique. La police des caractères, les couleurs, le fond d'écran choisi, sont autant de points faibles de certains sites. Le texte doit être clair, utile, non redondant, court (mais pas trop) et surtout d'actualité. La rédaction peut être de style télégraphique... la littérature, " c'est réservé au papier ». Les problèmes de lecture sont illustrés par la nécessité qu’ont certains étudiants de suivre " comme avec le doigt » les lignes du texte avec la flèche de la souris. Certains étudiants préferent travailler sur une version papier, sur laquelle ils peuvent intervenir physiquement en surlignant certains mots ou certains passages. Des étudiants proposent de fournir en fin de chapitre un résumé qui serait facile à imprimer afin de pouvoir garder une version courte sur papier, de l'enseignement.

\section{Linteractivité}

$\mathrm{Si}$, pour quelques étudiants, les liens hypertextes ne semblent pas indispensables, la majorité y est favorable. Le lien pourrait notamment donner accès à des notions de bases. Le lien pourrait permettre d'expliquer un mot, en 


\section{Nouvelles Technologies Éducatives}

donner une définition ou l'illustrer par une image ou une photographie afin de lui donner un sens. Le lien autorise l'accès à des notions complémentaires. Le lien avec une référence bibliographique étaye une affirmation, à condition de délivrer non seulement la référence proprement dite (auteurs, titre de l'article référencé, journal ou livre où figure le texte), mais aussi un résumé. Le lien pourrait donner accès à une version longue du texte qui n'apparấtrait pas en première intention. La plupart des étudiants évoquent le risque de se perdre dans le dédale des liens : le syndrome des Mille-et-une-nuits avec une lecture en tiroirs ou en " poupées russes " est redouté par beaucoup. De nombreux étudiants souhaitent pouvoir disposer de liens directs, de ponts, avec d'autres universités virtuelles pour ne pas perdre de temps à chercher les adresses de sites concernés par le même objet d'étude.

Cette exigence témoigne d'un véritable besoin de tutorat : les étudiants craignent d'être abandonnés vers un enseignement in abstentia qui ne peut pas remplacer, mais simplement compléter l'enseignement actuel. L'enseignement doit rester hybride, sans jamais oublier l'apprentissage au lit du malade ${ }^{13}$. Les étudiants proposent ainsi une sorte de plaidoyer pour plus d'humanisme dans la formation médicale ${ }^{14}$.

\section{Conclusion}

L'enseignement électronique est souhaité par les étudiants afin d'apporter un support visuel à l'enseignement traditionnel (illustrations, images, radiographies, animations, vidéo). Il est une source actualisée, récente d'informations remises à jour sans cesse, à l'opposé d'un livre qui doit attendre sa réédition pour être à jour. Il est une base de données utile pour la réalisation d'un travail de recherche. Le développement des connexions à haut débit et la miniaturisation de disques durs externes (clés USB) permettent le téléchargement de formations en ligne. Reste à proposer sur Internet un contenu de qualité utilisant à bon escient et avec une intention pédagogique claire les ressources proposées par les TIC. Pour que les TIC deviennent plus qu'une ressource informative mais un contenu pédagogique vrai, il faut que l'équipement informatique des étudiants progresse et que les enseignants améliorent l'usage qu'ils font des TIC à des fins pédagogiques.

Même si la disponibilité de l'outil informatique est encore inégale parmi les étudiants en médecine, les TIC ont pris une place importante en tant que ressource de connaissances.

\section{Contributions}

François Becmeur a conçu et réalisé l'étude et rédigé le manuscrit. Michèle Kirsch était directrice du travail de thèse dans lequel s'inscrit l'étude rapportée. Didier Mutter a apporté une aide méthodologique, a formulé des conseils et a participé à la relecture du manuscrit. Jacques Marescaux a formulé des conseils et participé à la relecture du manuscrit. 


\section{Annexe
Questionnaire distribué aux étudiants}

Age $\quad$ Sexe
Vous possédez un ordinateur : oui $\square \quad$ non $\square$
Vous utilisez un ordinateur (mais vous n'en possédé
celui de la Faculté $\square \quad$ celui d'un(e) ami(e)
Vous utilisez : un PC $\square \quad$ un Mac $\square \quad$ Jeux Vid
Autre
Vous utilisez des CD de formation ? oui $\square \quad$
Quel CD :
Revue de Médecine
CD distribué par un laboratoire $\square$
CD de congrès

Vous surfez sur Internet ? oui $\square$ non $\square$

Quel(s) site(s) visitez-vous le plus souvent?

Adresse(s) ?

$\mathrm{Ce}(\mathrm{s})$ site(s) sont-ils payants? oui $\square$ non $\square$

Si payant(s), dans quelle fourchette de prix ?

Si vous étiez conduit à entrer régulièrement sur un site (revue scientifique, site de formation, université virtuelle) grâce à un abonnement (annuel par exemple), combien seriez-vous prêt à dépenser par an ?

Vous souhaitez apprendre en langue française exclusivement ? oui $\square \quad$ non $\square$

Vous apprenez indifféremment en anglais ou en français? $\quad$ oui $\square \quad$ non $\square$

Mode de connexion ? Modem $\square$ ADSL $\square$ Cable $\square$

Autre (s)

Vous estimez être : un(e) utilisateur(trice) débutant (e) $\square \quad$ Initié(e) $\square \quad$ Expérimenté(e) $\square$ Vous utilisez des logiciels de :

$\begin{array}{ll}\text { traitement de texte } & \square \\ \text { présentation de diapositives } & \square \\ \text { des tableurs } & \square\end{array}$

Vous utilisez le courrier électronique ? oui $\square \quad$ non $\square$

Vos remarques à propos de l'intégration possible d'Internet dans votre formation: 


\section{Nouvelles Technologies Éducatives}

\section{Références}

1. Linard M. Des machines et des hommes. Apprendre avec les nouvelles technologies. Paris : Edition l'Harmattan, 1996.

2. Jacquinot G. Apprivoiser la distance et supprimer l'absence? Revue Française de Pédagogie 1993;102:5567.

3. Perriault J. La communication du savoir à distance. Paris : Edition l'Harmattan, 1996.

4. Lebrun M. Pédagogie et technologie en marche vers l'autrement. Pédagogie Médicale 2000;1:45-53.

5. Reding R, Denef JF, Parmentier P, Lebrun M. Accès, compétences et opinions des étudiants en médecine vis à vis des technologies de l'information et de la communication. Résultats d'une enquête auprès de 330 étudiants de l'UCL. Pédagogie Médicale 2001;2:242-9.

6. Becmeur F, Mutter D, Kirch M. Lettre à l'éditeur. Les nouvelles technologies de linformation et de la communication (NTIC) : un outil pédagogique pour étudiants en médecine? Pédagogie Médicale 2002;3:72-3.

7. Karsenti T. Conditions d'efficacité des formations ouvertes ou à distance (FOAD) en pédagogie universitaire. Pédagogie Médicale 2003;4:223-34.

8. Ricard JD, Lejoyeux M, El Ghoneimi A, Matheron S, Maillard D, Crickx B, Dreyfuss D. Utilisation des nouvelles technologies de l'information et de la communication par les étudiants en médecine. Enquête de pratique et mise en situation. Pédagogie Médicale 2005;6:112-22.
9. Quérin S, Tardif D, Messier IP, Ratelle R. Maîtrise de l'ordinateur et de l'information: une formation intégrée et continue au premier cycle des études médicales. Pédagogie Médicale 2001;2:108-13.

10. Projets de la LERU : EUREA et e-LERU [On-line]. Disponible sur :http://ulpmultimedia.ustrasbg.fr/ulpmultimedia2004/projetslerul

11. Denef JF. Evaluer les nouvelles technologies, oui, certes, mais comment ? Pédagogie Médicale 2003;4: 197-8.

12. Milgram S. Soumission à l'autorité. Paris :CalmannLévy, collection "Liberté de l'esprit", 1974.

13. Denef JF. Les nouvelles technologies de l'information et de la communication (NTIC) dans la formation médicale, au service de l'acquisition et du développement des compétences professionnelles. Pédagogie Médicale 2001;2:42-50.

14. Moulin D. L'apprentissage de l'interprétation médicale au coeur de la démarche clinique : un plaidoyer pour plus d'humanisme dans la formation médicale. Pédagogie Médicale 2005;6:5-6.

Manuscrit reçu le 17 mai 2005 ; commentaires éditoriaux adressés aux auteurs le 10 août 2005 ; accepté pour publication le 29 novembre 2005 